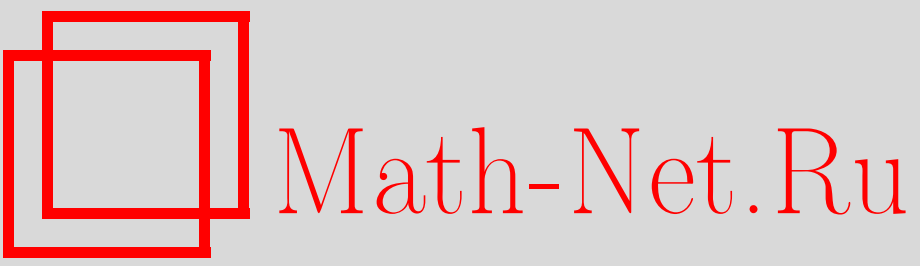

Д. У. Матрасулов, Волновые функции и энергетические уровни уравнения Шредингера с потенциалом, являющимся суммой потенциала двух кулоновских центров и потенциала гармонического осциллятора, ТМФ, 1998, том 117, номер 3, 364-369

DOI: https://doi.org/10.4213/tmf938

Использование Общероссийского математического портала Math-Net.Ru подразумевает, что вы прочитали и согласны с пользовательским соглашением

http://www.mathnet.ru/rus/agreement

Параметры загрузки:

IP: 54.224 .187 .69

26 апреля 2023 г., 18:16:48 
ТЕОРЕТИЧЕСКАЯ

И МАТЕМАТИЧЕСКАЯ

ФИЗИКА

Том 117, № 3

декабрь, 1998

(C) 1998 г.

Д.У. Матрасулов*

\section{ВОЛНОВЫЕ ФУНКЦИИ И ЭНЕРГЕТИЧЕСКИЕ УРОВНИ УРАВНЕНИЯ ШРЕДИНГЕРА С ПОТЕНЦИАЛОМ, ЯВЛЯЮШИМСЯ СУММОЙ ПОТЕНЦИАЛА ДВУХ КУЛОНОВСКИХ ЦЕНТРОВ И ПОТЕНЦИАЛА ГАРМОНИЧЕСКОГО ОСЦИЛЛЯТОРА}

Уравнение Шредингера для потенциала двух кулоновских центров плюс потенциал гармонического осциллятора решается методом эталонного уравнения при больших расстояниях между центрами. Получены асимптотические разложения для энергетических уровней и волновых функций.

\section{1. ВВЕДЕНИЕ}

Уравнение Шредингера с потенциалом двух кулоновских центров представляет значительный интерес с точки зрения различных задач, относяшихся к системам нескольких тел, рассматриваемым в приближении Борна-Оппенгеймера. Оно описывает связанные состояния легкой частицы в поле двух тяжелых частиц. Обычно такие системы встречаются в молекулярной физике. Однако в последние годы возник значительный интерес к другим системам подобного рода: моделям барионов с двумя тяжелыми кварками (QQq-барионы) [1] и моделям тяжелых гибридных мезонов с открытым флейвором (QQg-мезоны) [2]. В настоящее время эти системы становятся предметом широких исследований. В нерелятивистском приближении движение легкого кварка (глюона) в поле двух тяжелых кварков (пары кварк-антикварк) может быть описано уравнением Шредингера с потенциалом, который представляет собой сумму потенциала двух кулоновских центров и потенциала конфайнмента.

В данной работе рассматривается уравнение Шредингера с потенциалом, который является суммой потенциала двух кулоновских центров и потенциала гармонического осциллятора. Насколько нам известно, этот потенциал - единственный потенциал такого рода, допускающий разделение переменных. Используя метод эталонного уравнения, широко применяемый в молекулярной физике для решения уравнения Шредингера с потенциалом двух кулоновских центров, мы получаем собственные функции и собственные

\footnotetext{
*Отдел теплофизики Академии наук Республики Узбекистан, Ташкент, Узбекистан
} 
значения уравнения Шредингера с потенциалом двух кулоновских центров плюс потенциал гармонического осциллятора в виде асимптотических разложений по обратным степеням расстояния между центрами.

Итак, мы хотим найти собственные значения энергии и волновые функции следуюшего уравнения Шредингера:

$$
\left[-\frac{1}{2} \Delta-\frac{Z}{r_{1}}-\frac{Z}{r_{2}}+\omega^{2}\left(r_{1}^{2}+r_{2}^{2}\right)\right] \psi=E \psi .
$$

В вытянутых сфероидальных координатах, которые определяются как

$$
\xi=\frac{r_{1}+r_{2}}{R} \quad(1<\xi<\infty), \quad \eta=\frac{r_{1}-r_{2}}{R} \quad(-1<\eta<1),
$$

где $R$ - расстояние между кулоновскими центрами, этот потенциал можно записать в виде

$$
V\left(r_{1}, r_{2}\right)=-\frac{2}{R^{2}} \frac{a(\xi)+b(\eta)}{\xi^{2}-\eta^{2}}+\frac{\omega^{2} R^{2}}{2},
$$

здесь

$$
a(\xi)=2 Z R \xi-\frac{\omega^{2} R^{4}}{4} \xi^{2}\left(\xi^{2}-1\right), \quad b(\eta)=\frac{\omega^{2} R^{4}}{4} \eta^{2}\left(\eta^{2}-1\right) .
$$

Хорошо известно [3], что уравнение Шредингера с потенциалом вида (2) допускает разделение переменных в вытянутых сфероидальных координатах. После разделения переменных мы имеем три обыкновенных дифференциальных уравнения, связанных константами разделения $\lambda$ и $m$ :

$$
\begin{gathered}
{\left[\frac{\partial}{\partial \xi}\left(\xi^{2}-1\right) \frac{\partial}{\partial \xi}+a \xi+\left(p^{2}-\gamma^{\prime} \xi^{2}\right)\left(\xi^{2}-1\right)-\frac{m^{2}}{\left(\xi^{2}-1\right)}-\lambda\right] X(\xi)=0} \\
{\left[\frac{\partial}{\partial \eta}\left(\eta^{2}-1\right) \frac{\partial}{\partial \eta}+\left(p^{2}-\gamma^{\prime} \eta^{2}\right)\left(\eta^{2}-1\right)-\frac{m^{2}}{\left(\eta^{2}-1\right)}-\lambda\right] Y(\eta)=0} \\
\left(\frac{\partial^{2}}{\partial \phi^{2}}+m^{2}\right) Z(\phi)=0
\end{gathered}
$$

где

$$
\begin{aligned}
p & =\frac{R}{2} \sqrt{2 E^{\prime}}, & E^{\prime} & =E-\frac{\omega^{2} R^{2}}{2}, \\
\gamma^{\prime} & =\frac{\omega^{2} R^{4}}{4}, & a & =2 Z R .
\end{aligned}
$$

Подстановка

$$
\begin{aligned}
& U(\xi)=\frac{1}{\sqrt{\xi^{2}-1}} X(\xi), \\
& V(\eta)=\frac{1}{\sqrt{1-\eta^{2}}} Y(\eta)
\end{aligned}
$$


приводит эти уравнения к следующему каноническому виду:

$$
\begin{gathered}
U^{\prime \prime}(\xi)+\left[\frac{h^{2}}{4}+\frac{h(\alpha \xi-\lambda)}{\xi^{2}-1}-h^{4} \gamma \xi^{2}+\frac{1-m^{2}}{\left(\xi^{2}-1\right)^{2}}\right] U(\xi)=0 \\
V^{\prime \prime}(\eta)+\left[\frac{h^{2}}{4}+\frac{h \lambda}{1-\eta^{2}}-h^{4} \gamma \eta^{2}+\frac{1-m^{2}}{\left(1-\eta^{2}\right)^{2}}\right] V(\eta)=0
\end{gathered}
$$

где

$$
\alpha=2 Z / \sqrt{2 E^{\prime}}, \quad \gamma=\omega^{2} / 8 E^{\prime 2}, \quad h=2 p .
$$

Граничные условия для функций $U$ и $V$ имеют вид

$$
\begin{aligned}
\left.U(\xi)\right|_{\xi=1} & =0,\left.\quad U(\xi)\right|_{\xi \rightarrow \infty} \longrightarrow 0 \\
\left.V(\eta)\right|_{\eta= \pm 1} & =0
\end{aligned}
$$

\section{2. АСИМПТОТИЧЕСКИЕ РЕШЕНИЯ "КВАЗИУГЛОВОГО" УРАВНЕНИЯ}

Мы решаем уравнения (3) и (4) приближенно при больших $R$ методом эталонного уравнения. Этот метод успешно применялся при решении нерелятивистской задачи двух кулоновских центров [3-5] и в теории дифракции волн. Детали метода эталонного уравнения приведены в [3-6]. Начнем с “углового” уравнения (4). В качестве эталонного уравнения для (4) мы выбираем уравнение Уиттэкера [7]

$$
W^{\prime \prime}+\left[-\frac{h^{4}}{4}+\frac{h^{2} k}{z}+\frac{1-m^{2}}{4 z^{2}}\right] W=0
$$

и ишем решение в виде

$$
V=\left[z^{\prime}(\eta)\right]^{-\frac{1}{2}} M_{k, \frac{m}{2}}\left(h^{2} z\right),
$$

где $M_{k, m / 2}\left(h^{2} z\right)$ есть регулярное в нуле решение уравнения (8). После подстановки (9) в (4) получаем следующее уравнение для $z$ :

$$
\begin{array}{r}
\frac{z^{\prime 2}}{4}-\gamma(x-1)^{2}-\frac{1}{h^{2}}\left(\frac{1}{4}+\frac{k z^{\prime 2}}{z}-\frac{\lambda}{2 x\left(1-\frac{x}{2}\right)}\right)+ \\
+\frac{\tau}{h^{2}}\left(\frac{1}{x^{2}\left(1-x^{2}\right)}-\frac{z^{\prime 2}}{z^{2}}\right)-\frac{1}{2 h^{2}}[z, x]=0
\end{array}
$$

где $\tau=\left(1-m^{2}\right) / 4, x=1+\eta,[z, x]=-(3 / 2)\left(z^{\prime \prime} / z^{\prime}\right)^{2}+z^{\prime \prime \prime} / z^{\prime}\left(z^{\prime}-\right.$ производная $z$ по $\left.x\right)$. Требование совпадения переходных точек

$$
\left.z(x)\right|_{x=0}=0
$$

приводит к следующему “квантовому условию":

$$
\lambda=2 k z^{\prime}(0)+\frac{2 \tau}{h^{2}}\left[\frac{z^{\prime \prime}(0)}{z^{\prime}(0)}-1\right] .
$$


Будем искать решение уравнения (10) и собственные значения $\lambda$ в виде следующих асимптотических разложений:

$$
z=\sum_{k=0}^{\infty} \frac{z_{k}}{h^{k}}, \quad \lambda=\sum_{k=0}^{\infty} \frac{\lambda_{k}}{h^{k}} .
$$

Подстановка этих разложений в (10) дает рекуррентную систему дифференциальных уравнений для $z$ :

$$
\begin{aligned}
& z_{0}^{\prime}=2 \gamma^{\frac{1}{2}}(x-1), \\
& z_{1}^{\prime}=0 \\
& z_{2}^{\prime}=\frac{1}{2 z_{0}^{\prime}}+\frac{2 k z_{0}^{\prime}}{z_{0}}-\frac{\left(z_{1}^{\prime}\right)^{2}}{2 z_{0}^{\prime}}-\frac{2 \lambda_{0}}{z_{0}^{\prime} x(1-x / 2)}-\frac{z_{2}^{\prime 2}}{2},
\end{aligned}
$$

и рекуррентные соотношения для $\lambda$ :

$$
\begin{aligned}
& \lambda_{0}=2 k z_{0}^{\prime}(0), \\
& \lambda_{1}=2 k z_{1}^{\prime}(0), \\
& \lambda_{2}=2 k z_{2}^{\prime}(0)+2 \tau\left(\frac{z_{0}^{\prime \prime}(0)}{z_{1}^{\prime}(0)}-1\right),
\end{aligned}
$$

Решая эти рекуррентные уравнения, имеем

$$
\begin{aligned}
\lambda^{(\eta)} & =4 k \gamma^{\frac{1}{2}}+\frac{2 k \beta-4 \tau}{h^{2}}+O\left(\frac{1}{h^{4}}\right), \\
z & =\gamma^{\frac{1}{2}} x(2-x)+\frac{1}{h^{2}} \beta \ln (1-x)+O\left(\frac{1}{h^{4}}\right) .
\end{aligned}
$$

Из граничных условий можно получить следующее выражение для квантового числа $k[5,6]:$

$$
k=q+\frac{m+1}{2}
$$

где $q=0,1,2, \ldots$

\section{3. АСИМПТОТИЧЕСКИЕ РЕШЕНИЯ КВАЗИРАДИАЛЬНОГО УРАВНЕНИЯ}

В качестве эталонного уравнения для (3) возьмем уравнение

$$
W^{\prime \prime}+\left[h^{2} s-h^{4} y^{2}-\frac{4 \tau+3}{4 y^{2}}\right] W=0,
$$

решения которого выражаются через вырожденные гипергеометрические функции $[1,7]$

$$
W=y^{c} \exp \left\{-\frac{h^{4} y^{2}}{2}\right\} F\left(\frac{s-2 c-1}{4}, c+\frac{1}{2}, h^{4} y^{2}\right)
$$


где

$$
c=\frac{1+\sqrt{m^{2}+3}}{2} .
$$

Граничные условия (6) и свойства функций $F[7,8]$ дают $s=4 n+\sqrt{m^{2}+3}+2$. Подставляя

$$
U=[y(\xi)]^{-\frac{1}{2}} W(y(\xi))
$$

в уравнение (3), получим

$$
\begin{gathered}
\frac{y^{2} y^{\prime 2}}{4}-\gamma \xi^{2}+\frac{1}{h^{2}}\left(\frac{1}{4}-s y^{2}-\frac{\lambda}{\xi^{2}-1}\right)+\frac{1}{h^{3}} \frac{\alpha \xi}{\xi^{2}-1}+ \\
+\frac{4 \tau}{h^{4}\left(\xi^{2}-1\right)^{2}}-\frac{3-4 \tau}{4 h^{4}} \frac{y^{\prime 2}}{y^{2}}-\frac{1}{2 h^{4}}[y, \xi]=0 .
\end{gathered}
$$

После подстановки $\phi=y^{2}(t) / 4$ это уравнение можно привести к виду

$$
\begin{gathered}
\phi^{2}-\gamma(t+1)+\frac{1}{h^{2}}\left(\frac{1}{4}-\left(n+\frac{1}{2}\right) \frac{\phi^{\prime 2}}{\phi}-\frac{\lambda}{t(t+2)}\right)+\frac{1}{h^{3}} \frac{\alpha(t+1)}{t(t+2)}+ \\
+\frac{\tau}{h^{4}}\left(\frac{\phi^{\prime 2}}{\phi^{2}}-\frac{4}{t^{2}(t+2)^{2}}\right)-[\phi, t]=0
\end{gathered}
$$

где $t=\xi-1$. Условие квантования, которое следует из условия $\phi(t)=0$, записьвается в виде

$$
\lambda=-2 s \phi^{\prime}(0)+\frac{\alpha}{h}-\left.\frac{1}{h^{2}}\left[\frac{\phi^{\prime \prime}}{\phi^{\prime}}+1\right]\right|_{t=0} .
$$

Подставляя в уравнение (16) асимптотические выражения

$$
\phi=\sum_{k=0}^{\infty} \frac{\phi_{k}}{h^{k}}, \quad \lambda=\sum_{k=0}^{\infty} \frac{\lambda_{k}}{h^{k}}
$$

и решая полученные уравнения, имеем

$$
\begin{aligned}
y= & 2 \gamma^{\frac{1}{4}}\left(t^{2}+2 t\right)^{\frac{1}{2}}+\frac{1}{h^{2}} \delta \gamma^{-\frac{1}{4}}\left(t^{2}+2 t\right)^{-\frac{1}{2}} \ln (t+1)+ \\
& +\frac{1}{h^{3}} \alpha \gamma^{-\frac{3}{4}}\left(t^{2}+2 t\right)^{-\frac{1}{2}} \ln \frac{2(t+1)}{t+1}+O\left(\frac{1}{h^{4}}\right), \\
\lambda^{(\xi)}= & -2 s \gamma^{\frac{1}{2}}-\frac{\alpha}{h}+\frac{4 \tau-s \delta}{h^{2}}-\frac{s \alpha \gamma^{-\frac{1}{4}}}{2 h^{3}}+O\left(\frac{1}{h^{4}}\right) .
\end{aligned}
$$

\section{4. АСИМПТОТИЧЕСКИЕ РАЗЛОЖКНИЯ ДЛЯ ЭНЕРГИИ И ВОЛНОВЫХ ФУНКЦИЙ}

Асимптотические разложения (12) и (19) дают выражение для энергии в виде мультипольного разложения. Чтобы получить это разложение, нужно подставить выражение

$$
E^{\prime}=E_{0}+\frac{E_{1}}{R}+\frac{E_{2}}{R^{2}}+\cdots
$$


в разложения (12) и (19). Приравнивая $\lambda^{(\eta)}$ и $\lambda^{(\xi)}$ и учитывая равенства $(5)$, получаем следующие выражения для коэффищиентов $E_{1}, E_{2}, \ldots$ :

$$
\begin{aligned}
& E_{1}=\frac{1}{6 Z}\left[\left(s \omega-2 k \omega^{-1}\right)\left(2 E_{0}\right)^{\frac{5}{2}}+\left(4 s^{2}-16 k^{2}-16 \tau\right)\left(2 E_{0}\right)^{\frac{3}{2}}\right], \\
& E_{2}=\frac{5}{2} E_{1}^{2}+2 s \omega^{-1} E_{0}+E_{1}\left(2 E_{0}\right)^{\frac{1}{2}} Z^{-1}\left(16 \tau^{2}+16 k^{2}-4 s^{2}\right),
\end{aligned}
$$

Теперь необходимо найти $E_{0}$. Для этого заметим, что при $R \rightarrow \infty$ имеем $E^{\prime}=E_{0}$ или

$$
E=E_{0}+\frac{\omega^{2} R^{2}}{2}
$$

С другой стороны, при больших $R$

$$
\begin{aligned}
V\left(r_{1}, r_{2}\right)= & \frac{2 Z}{R} \sum_{l=0}^{\infty}\left(\frac{r}{R}\right)^{l} P_{l}(\cos \theta)+\omega^{2}\left[\left(r^{2}+2 r R \cos \theta+\frac{R^{2}}{4}\right)+\right. \\
& \left.+\left(r^{2}-2 r R \cos \theta+\frac{R^{2}}{4}\right)\right] \approx \omega^{2}\left(2 r^{2}+\frac{R^{2}}{2}\right) .
\end{aligned}
$$

Для такого потенциала энергетические уровни имеют вид

$$
E=2 \omega\left(N+\frac{3}{2}\right)+\frac{\omega^{2} R^{2}}{2},
$$

где $N=2 n+q+m$ есть главное квантовое число. Сравнивая формулы (20) и (22), видим, что

$$
E_{0}=2 \omega\left(N+\frac{3}{2}\right) .
$$

Таким образом, получены асимптотические разложения для волновых функций и собственных значений энергий уравнения Шредингера с потенциалом, представляющим собой сумму потенциала двух кулоновских центров и потенциала гармонического осциллятора. Полученные формулы могут оказаться полезными при дальнейших численных расчетах, а также могут быть использованы для оценки энергетических спектров QQq-барионов.

\section{Список литературы}

[1] J. M. Richard. Phys. Rep. 1992. V. 212. P. 1

[2] S. N. Mukherjee, R. Nag, S. Sanyal, T. Morii, J. Morishita, M. Tsuge. Phys. Rep. 1993. V. 231. P. 203.

[3] В. И. Комаров, Л. И. Пономарев, Ю. С. Славянов. Сфероидальные и кулоновские сфероидальные функции. М.: Наука, 1978.

[4] Ю.С. Славянов. Асимптотики одномерного уравнения Шредингера. Л.: Изд-во Ленингр. университета, 1991

[5] В. И. Комаров, Ю. С. Славянов. ЖЭТФ. 1967. Т. 52. С. 1368.

[6] V. I. Komarov, Yu. S. Slavyanov. J. Phys. B. 1968. V. 1. P. 1066.

[7] Г. Бейтмен, А. Эрдейи. Высшие трансцендентные функции. Т. 1. М.: Наука, 1974.

[8] М. Абрамовии, И. Стиган (ред.). Справочник по специальным функциям с формулами, графиками и математическими таблицами. М.: Наука, 1979.

Поступила в редакцию 13.IV.1998 г.

2 Теоретическая и математическая физика, т. 117, № 3, 1998 г. 